\title{
Priority setting in the NHS: reports from six districts
}

\author{
Chris Ham
}

This paper is based on a speech given at the conference on Priority Setting in the Health Service, London, March 1993. $A$ collection of papers from the conference will be published in book form later this year.

\begin{abstract}
District health authorities must make choices on the use of their funds and the priorities that should be pursued. In future they may be able to place greater weight on the views of local people and evidence on cost effectiveness of different services. This paper reviews developments in a recent study of six district health authorities with an interest in priority setting. On the whole they had avoided excluding services entirely from their contracts; they experienced difficulty in comparing different services, and made more progress by analysing priorities within individual service areas than by comparing quite different services. The absence of information to guide priority setting was a major problem. A major effort had been made to involve the public in decision making, and a range of tools and methods developed to support work on priority setting. The impact of the work done so far has varied; district health authorities are still at an early stage in developing their work on priority setting.
\end{abstract}

The establishment of district health authorities as purchasers of health services for their residents is a key element in the NHS reforms. In their role as purchasers, district health authorities are responsible for assessing the population's need for health care and deciding which services to buy to meet this need. Operating within cash limited budgets determined by regional health authorities, district health authorities have to agree priorities for the use of the resources they have at their disposal. In part these priorities are intended to reflect policies laid down by ministers and regional health authorities, and in part they are meant to be a response to the assessment of needs carried out locally by district health authorities. Given that not everything can be done within the available resources, district health authorities are therefore in the position of making choices on the use of their funds and the priorities that should be pursued.

This responsibility is not new to district health authorities, who have long been in the position of having to determine local priorities for service development. What is different is that, as purchasers, district health authorities may be able to take decisions which depend less on the demands of providers than they have in the past. In so doing, district health authorities should be able to place greater weight on other factors, such as the views of local people and evidence on the cost effectiveness of different services.

In simplified form, the figure shows the situation in which district health authorities find themselves. If, in the past, factors along the vertical axis, represented by national and regional "givens" and the demands of local professional interests, have been particularly influential, in the future there is an opportunity to attach greater emphasis to public views and research evidence. To underscore the importance of the horizontal axis, experience in Oregon suggests that it was precisely this combination of public opinion and analysis of data drawn from the scientific literature that influenced the decisions of the Health Services Commission, set up to determine priorities for the use of Medicaid funds.

How have district health authorities responded to the challenge of priority setting? A recent study of six authorities that were known to have taken a particular interest in priority setting contains some early indications of likely developments. The study reviewed experience in the City and Hackney, Mid Essex, Oxfordshire, Solihull, Southampton and South West Hampshire, and Wandsworth up to December 1992. Interviews were conducted with those most closely involved in priority setting, and relevant papers and reports were analysed. The aim was to describe the approach taken in each district, evaluate the lessons that had emerged, and identify implications for the future. The rest of this paper summarises the results of the study, and the full report will be available shortly. ${ }^{2}$

\section{Findings from six districts}

The district health authorities in the study had on the whole avoided excluding services entirely from their contracts. This is important because much of the public debate about priority setting has centred on districts where services like tattoo removal and in vitro fertilisation are no longer funded by health authorities. In place of exclusions, there was growing interest in the development of guidelines on which patients are most likely to benefit from particular treatments. This is discussed further below.

A second finding was that district health authorities experienced difficulty in comparing quite different services. This is because there is no common currency for making these broad comparisons. Weighing the priority that should be given to health promotion as against additional operations to reduce waiting times for surgery or higher quality of care for people with learning difficulties is like comparing apples, oranges, and kiwi fruit. At present, there is no reliable basis on which to make these judgments, although over time the development of league tables for cost per QALY (quality adjusted life year) may provide an aid to decision making. In this study, there was very little evidence that the research on QALYs had exerted any

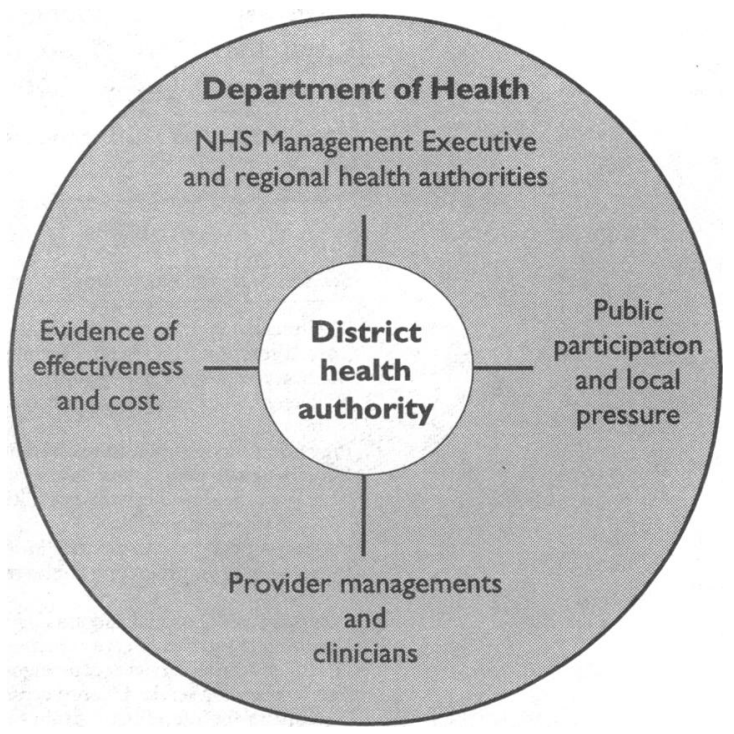

District health authorities find themselves caught between national and regional "givens" and demands of local professional interests (vertical axis) and public views and research evidence (horizontal axis) ${ }^{1}$ 
influence on district health authorities. Indeed, there seems to be a considerable gap between the work of health economists in this area and the world in which purchasers operate.

Linked to this, a third issue is that district health authorities were able to make more progress by analysing priorities within individual service areas or disease categories than by comparing different services. One example of this was found in Oxfordshire, where the authority was seeking to examine the scope for redistributing resources within the existing envelope of expenditure allocated to individual service areas so that it could obtain additional health gain. For example, in the case of heart disease, it was considering spending less on inpatient admission for myocardial infarction and more on coronary rehabilitation.

Another example was the approach taken in Wandsworth to review the use of resources for the prevention and treatment of diabetes. In this case, a health economist employed by the health authority had shown how a large volume of resources was spent on a small number of patients. These patients had multiple health and social problems and were admitted to hospital for lengthy stays. Much less money was spent on a large number of patients looked after by general practitioners and diabetologists. This type of analysis, involving the examination of priorities in terms of "bite sized chunks," showed the potential for using resources more effectively within individual disease categories.

A fourth finding, and one that is particularly significant, is that the absence of information to guide priority setting was perceived as a major problem everywhere. In particular, information on the cost effectiveness of services was often lacking or incomplete. The effectiveness bulletins put out by the universities of Leeds and York had started to fill some of the gaps that exist and were welcomed by those involved in purchasing. ${ }^{3}$ But there was a view that much more could be done to summarise the results of research on the effectiveness of different interventions and to present this in a form that could be readily assimilated by busy managers and their colleagues.

Fifthly, a major effort had been made to involve the public in decision making. One of the reasons for this is that district health authorities recognise that priority setting cannot be reduced to a technical or scientific exercise. Inevitably, the process of setting priorities involves making judgments on the basis of incomplete information and evidence. These judgments are likely to be more soundly based and defensible if they have been exposed to public discussion. In other words, given that there is no right answer in the priority setting debate, an important justification for the decisions that are made is that they have been arrived at as a result of due process.

A variety of methods has been used to involve the public in decision making. For example, in Solihull the district health authority has undertaken a survey of public attitudes to elicit views on the value of different health states and on priorities for service development. This was done through a questionnaire survey of 600 adults.

The City and Hackney district health authority has also sought the public's views through a questionnaire survey. This was supplemented by meetings with community groups, at which 350 people were interviewed, and by open public meetings. The public's rankings of 16 services were compared with the priorities expressed by general practitioners, consultants, and public health doctors (table). As the table shows, the public's views in some cases were appreciably different from those of the medical respondents.

Another important finding from the City and Hackney project was that the public's assessment of priorities depended critically on the wording of the questions put to them. As an example, intensive care for premature babies was ranked highly when the survey questionnaire was piloted. Subsequently, when the question was qualified with the statement "babies weighing less than one and a half pounds are unlikely to survive," its ranking dropped considerably. This has important implications for the design of questionnaires and the methods used to understand the public's views.

A different approach to public participation has been pursued in Mid Essex Health Authority. A number of methods have been used in Mid Essex, including collaboration with the community health council, working with voluntary organisations to discuss and rank priorities for service developments, and making use of the rapid appraisal technique in one part of the district to gather data about the community's views and values. Information from these different sources has been used by the health authority to determine how to allocate its growth funds. In 1992, this consisted of giving particular priority to mental health services.

\section{TOOLS FOR PRIORITY SETTING}

A sixth finding was that a wide range of tools and methods have been developed to support work on priority setting. Again, Mid Essex exemplifies this, having used a variety of tools to identify priorities. These include a device known as 16 health care building blocks, a list of priorities originally developed by the BBC's Public Eye documentary programme, and a similar exercise prepared in the district which has

Main results of a public priorities survey in City and Hackney Health Authority, 1992. Values are mean priority ranks (1-highest priority, $16=$ lowest)

\begin{tabular}{|c|c|c|c|c|}
\hline Services or treatments (examples) & $\begin{array}{c}\text { Public } \\
(n=335)\end{array}$ & $\begin{array}{c}\text { General } \\
\text { practitioners } \\
(n=66)\end{array}$ & $\begin{array}{c}\text { Consultants } \\
(\mathrm{n}=116)\end{array}$ & $\begin{array}{l}\text { Public health } \\
\text { doctors } \\
(n=6)^{\star}\end{array}$ \\
\hline Treatments for children with life threatening illness (leukaemia) & 1 & 5 & 2 & 9 \\
\hline Special care and pain relief for people who are dying (hospice care) & 2 & 4 & 4 & 8 \\
\hline Medical research for new treatments & 3 & 11 & 8 & 11 \\
\hline \multicolumn{5}{|l|}{ High technology surgery and procedures which treat life threatening conditions (heart or liver } \\
\hline transplants) & 4 & 12 & 12 & 12 \\
\hline Preventive services (screening, immunisation) & 5 & 6 & 7 & 4 \\
\hline Surgery to help people with disabilities to carry out everyday tasks (hip replacements) & 6 & 8 & 5 & 5 (equal) \\
\hline \multicolumn{5}{|l|}{ Therapy to help people with disabilities carry out everyday tasks (speech therapy, physiotherapy, } \\
\hline Services for people with mental illness (psychiatric wards, community psychiatric nurses) & 8 & 2 & 1 & 1 (equal) \\
\hline \multirow{2}{*}{\multicolumn{5}{|c|}{$\begin{array}{l}\text { Intensive care for premature babies who weigh less than one and a half pounds and are unlikely } \\
\text { to survive }\end{array}$}} \\
\hline & 9 & 13 & 13 & 15 (equal) \\
\hline Long stay care (hospital and nursing home for elderly people) & 10 & 3 & 6 & 10 \\
\hline Community services or care at home (district nurses) & 11 & 1 & 3 & 1 (equal) \\
\hline Health education services (campaigns encouraging healthy lifestyles) & 12 & 10 & 11 & 5 (equal) \\
\hline Family planning services (contraception) & 13 & 9 & 9 & 1 (equal) \\
\hline Treatments for infertility (test tube babies) & 14 & 14 & 14 & 15 (equal) \\
\hline Complementary or alternative medicine (acupuncture, homoeopathy, herbalism) & 15 & 15 & 16 & 13 (equal) \\
\hline Cosmetic surgery (tattoo removal, removal of disfiguring lumps and bumps) & 16 & 16 & 15 & 13 (equal) \\
\hline
\end{tabular}

* Little can be construed from this sample as numbers were very small. 
been used to rank 12 services in order of priority. The purpose of all of these tools is to explore with a variety of interests (general practitioners, community health councils, voluntary organisations, etc) the relative priority attached to different services.

In City and Hackney, as well as research to investigate how the public viewed priorities, the Director of Public Health has developed a two stage scoring method to help inform priority setting. In the first stage, bids are ranked on the basis of needs assessment, with greatest weight being attached to services which respond to local needs. In the second stage, after a shortlist has been prepared, individual proposals are ranked by using the following criteria:

- Robustness or implementability of proposal (scored 0-3)

- Promotion of equity (0-1)

- Evidence of effectiveness or cost effectiveness $(0 \cdot 2)$

- Collaboration with or integration with primary care (0-3)

- Prioritised by community health council (0-1)

- Prioritised by general practitioner or general practitioners' forum (0-1)

- Other possible or more appropriate sources of funding $(0-5$; negative score).

The greatest weight is therefore attached to whether other funding methods are available, using a negative score as high as five to cancel out the other weightings.

A similar method is used in Wandsworth. In 1992-3, proposals were ranked by using the following criteria and weightings, up to a possible score of 100 :

- Gives potential for health gain (40)

- Improves quality of service (20)

- Accords with local views (20)

- Achievable in current year (15)

- Accords with national and regional priorities (5).

In this approach, potential for health gain is an all embracing factor encompassing length of life as well as quality. The ranking exercise was undertaken among both the purchasing team and the non-executive members of the district health authority, and an agreed list of priorities was drawn up at the end of the exercise.

\section{Impact}

One of the questions that inevitably arises from the work done so far is, what impact has it had on decision making? In some districts, of which Solihull is probably the best example, the answer is that there has so far been a very limited impact. This is largely because the work carried out in Solihull was still being analysed at the time when the research was done and it would be premature to expect major changes to have occurred.

In other districts, and here Mid Essex is a good example, it is possible to point to decisions that have been shaped by the interest shown in priority setting. The decision to give higher priority to mental health services was a direct reflection of the discussions that took place locally with key interests. Similarly, in City and Hackney the district health authority decided to protect community services in the face of considerable pressure to allocate extra resources to acute services.

In the remaining districts, the impact of the work done has been real but less tangible. Managers in these districts refer to a change in the culture of their organisations and a new approach to thinking about priorities without being able to cite specific examples of decisions that have been altered. No doubt this is partly because of the interest shown in exercises and simulations of priority setting in districts like Oxfordshire, Southampton and South West Hampshire, and Wandsworth. It is also because some of the analysis has been research based and was not designed with the aim of directly influencing purchasing decisions at this stage.

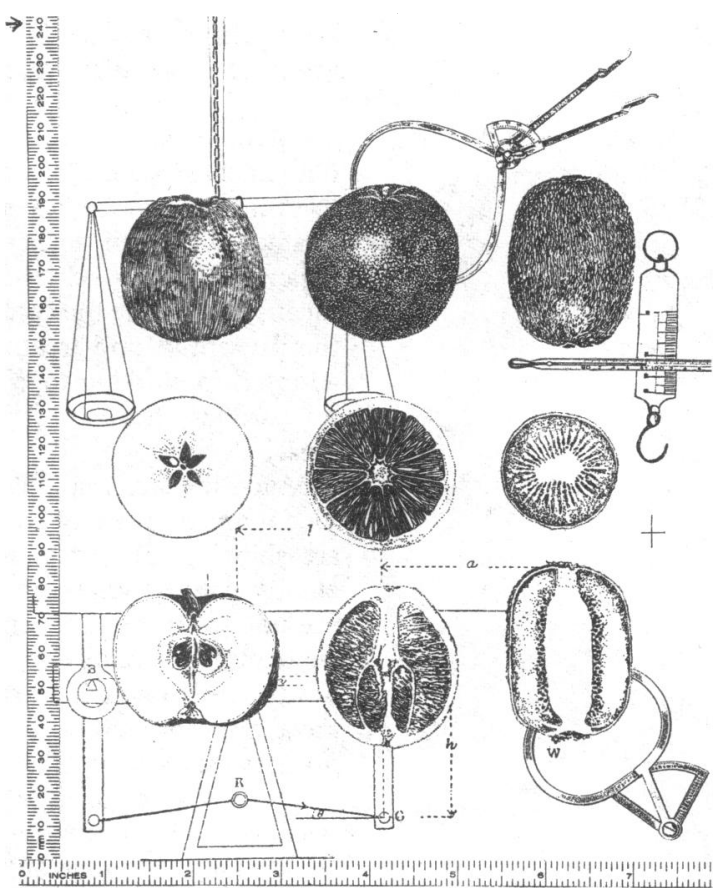

Weighing the priority that should be given to health promotion as against additional operations to reduce waiting times for surgery or higher quality of care for people with learning difficulties is like comparing apples, oranges, and kiwi fruit

Despite this it is possible to show that the investment of time and effort has made a difference. Examples include Oxfordshire, where agreement has been reached on the values that shape purchasing, and Southampton and South West Hampshire, where the emphasis placed on widespread consultation on priorities illustrates how the process of purchasing has been influenced. This in turn has helped to frame the strategic priorities set out in the purchasing plan. Similarly, in Wandsworth the development of criteria for ranking bids for development has made more explicit the basis on which decisions are reached.

\section{Emerging issues}

The conclusion to emerge from this study is that district health authorities are still at an early stage in the development of their work on priority setting. The six districts whose work has been studied were chosen because they were known to be particularly interested in the process of priority setting. As such, they could make some claim to being at the leading edge of development in this particular area. Despite this it is clear that only limited progress has been made to date.

It is also apparent that a good deal of innovation is occurring in these districts. In the absence of a national strategy on the part of the government to set priorities for the NHS as a whole, district health authorities have taken the lead in developing approaches which they believe are appropriate. This makes it difficult to generalise about the work that has been done so far because a wide range of initiatives has been launched. As further experience is gained it will be important to find a way of sharing local knowledge to enable other health authorities to learn from the work that has been done.

On the basis of this study it is apparent that district health authorities could be assisted in the work they are doing if there were a more concerted approach nationally to draw together information on effectiveness and cost effectiveness in a usable form. Although the effectiveness bulletins produced by the universities of Leeds and York are seen as useful, it is not clear why the topics they cover have been chosen (glue ear and screening for osteoporosis are hardly at the top of the 
NHS agenda) and it would help purchasers if data were available in relation to services which are clearly at the centre of government policy. Nowhere is this more important than in relation to The Health of the Nation. District health authorities have no choice but to pursue the objectives set out in the national health strategy, but they do have discretion in selecting the means for their achievement. At its simplest, this means identifying the comparative costs and benefits of the preventive and treatment strategies that might be adopted in working towards the government's targets for coronary heart disease, cancer, and other priority areas.

Much the same applies in relation to the shift from secondary to primary care. District health authorities are showing interest in this issue as they work more closely with general practitioners. What is lacking at present is information and evidence that summarises views on best practice to enable district health authorities to decide which kind of services can be provided most appropriately and effectively in and around a primary care setting. This includes not only issues such as the treatment of asthma and diabetes but also the role of specialists in doing some of their outpatient work alongside general practitioners, the scope for extending minor surgery and investigations in primary care, and the use of staff such as physiotherapists, dietitians, and counsellors in general practice. There was a strong feeling among those interviewed that much of this information is available but it has yet to percolate through to those responsible for developing purchasing plans.

One approach worth examining is the work done in Wales to produce protocols for investment in health gain. The stated purpose of these protocols is to discern areas where further investment would bring worthwhile health gain. Topics covered include cancer, cardiovascular disease, maternal and early child health, and physical and sensory disabilities. Drawing on research findings and expert views, each protocol provides an assessment of need and identifies options for meeting this need through changes in priorities or service delivery. As such, published reports provide a resource for use by health authorities. While elements of this approach exist in England (for example, the needs assessment work commissioned by the NHS Management Executive) there is no comparable work which brings together information on needs and service effectiveness in a form that is accessible to health authorities.

In this context, the research undertaken by James Raftery seems to offer a way forward. This research combines a disease MAPping approach with evidence on cost effectiveness. Disease MAPs-Measurements of Activity and Price-provide information on the numbers of people suffering from particular diseases and on associated patterns of expenditure. Drawing on available data on cost effectiveness and making use of comparative health service indicators, Raftery has matched the use of health service facilities to the resources consumed. This approach (unpublished data) illustrates how this approach can be applied to support work on priority setting for health gain.

\section{Future developments}

In emphasising the need to provide better information to support work on priority setting, the importance of other inputs to decision making should not be neglected. Above all, further work is needed to establish the most appropriate ways of involving the public in decision making. The experience of $\mathrm{City}$ and Hackney Health Authority serves as a cautionary tale. The way in which questions are framed seems to influence the responses that are given. Any attempt to seek the public's views needs to incorporate this lesson. Indeed, given the complexity of some of the choices that have to be made, it may be that an investment in informing and educating the public about the issues involved is needed before citizens are asked to list priorities in rank order.

To return to an earlier point, one particularly promising path for further development is the use of guidelines to identify those patients who are most likely to benefit from particular interventions. The value of guidelines has been acknowledged by a committee set up by the Dutch government to examine choices in health care. The Dunning committee argued that guidelines should be drawn up by professional and scientific bodies as one part of a strategy for approaching priority setting. ${ }^{4}$ In particular, guidelines were seen to be important in ensuring the appropriate use of services.

In this context, appropriateness refers both to the selection of patients (restricting treatment to those patients who can clearly benefit) and to the care setting (home care, primary care, outpatient, day patient, and inpatient care). The underlying argument here is that a focus on appropriateness should enable better use to be made of available resources. This is a central thrust of the approach adopted by general practitioner fundholders, who maintain that they are achieving significant improvements in services through delivering these services more appropriately. The existence of wide variations in clinical practices ${ }^{5}$ suggests that there is considerable scope for change in this respect.

An unanswered question is whether the greater use of guidelines will obviate the need to define more precisely the boundaries of NHS provision. More specifically, would it be necessary to exclude a wider range of services from the NHS? At the time of writing there are conflicting indications on this point. On the one hand, it would seem that some services, such as long term nursing care, are increasingly being excluded in many places, and others (dental care for adults, for example) may be added. On the other hand, there is the experience of New Zealand, where a committee set up by the government in 1992 to identify core services to be available in the public health scheme concluded that this was not best done by drawing up a list of such services. As the committee noted, the starting point of any analysis should be that existing services reflected "decades of reasonably commonsense and principled decision making." As such, these services could be said to constitute the core of service provision.

Like the Dunning committee, the New Zealand core services committee went on to argue that greater attention should be paid to the appropriate use of all services. To assist in this process it initiated a series of consensus conferences to develop guidelines for using specific interventions. As the debate about priority setting develops in the United Kingdom, it is this focus on what were earlier referred to as bite sized chunks of service provision that seems to hold out most promise.

This paper is based on research carried out in association with Frank Honigsbaum and David Thompson. I thank them for their assistance. Thanks are also due to the Research and Development Directorate in the NHS Management Executive, which supported this research.

\footnotetext{
1 Heginbotham C, Ham C. Purchasing dilemmas. London: King's Fund College, 1992.
}

2 Ham C, Honigsbaum F, Thompson D. Priority setting for health gain. London: NHS Management Executive (in press).

3 University of Leeds; University of York. Effective health care. Leeds: The Universities, 1992, 1993.

4 Ministry of Welfare, Health, and Cultural Affairs. Choices in health care. Ministry of Welfare, Health, and Cultural Affar
Rijswijk: The Ministry, 1992. (Dunning report.)

5 Ham C, ed. Health care variations. London: King's Fund Institute, 1988 6 National Advisory Committee. Core services 1993/4. Wellington: NAC, 1992. 